\title{
Tunneling-induced deformation of bare frame structures on sand: a numerical study of building deformations
}

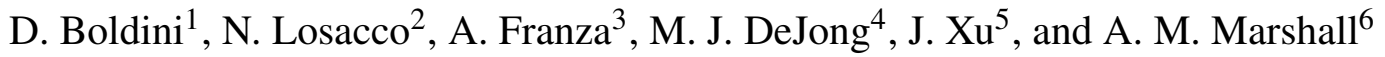 \\ ${ }^{1}$ Dept. of Chemical Engineering Materials Environment, Sapienza University of Rome, Rome, Italy. \\ ${ }^{2}$ Dept. of Civil, Environmental, Land, Building Eng. and Chemistry, Polytechnic University of Bari, Bari, Italy \\ ${ }^{3}$ Dept. of Civil and Architectural Engineering, Aarhus University, Aarhus, Denmark. \\ ${ }^{4}$ Dept. of Civil and Environmental Engineering, University of California, Berkeley, USA. \\ ${ }^{5}$ Institute of Geotechnical Engineering, School of Transportation, Southeast University, Nanjing, China. Formerly, \\ Dept. of Civil Engineering, University of Nottingham, Nottingham, UK. \\ ${ }^{6}$ Dept. of Civil Engineering, University of Nottingham, Nottingham, UK.
}

\section{ABSTRACT}

The paper compares the performance of two Finite Element Method approaches in reproducing the response of bare frame structures to tunneling in dry dense sand. A fully coupled approach, in which the tunnel, frame and soil are accounted for, is compared with a two-stage method incorporating simpler structural and soil models. The two approaches are validated against centrifuge test results of tunneling in sand beneath frames founded on either rafts or separate footings. Both approaches provide good estimates of displacements and distortions experienced by the frames provided that the soil-foundation interface and structural stiffness are correctly accounted for. The numerical models are also employed to extend the range of eccentric configurations investigated with centrifuge tests. The results demonstrate that shear deformations play an important role for all considered buildings, whereas only frames on separate footings are sensitive to horizontal ground movements. Finally, data are synthesized using modification factors and recently proposed relative stiffness terms.

\section{INTRODUCTION}

The increasing need for efficient and high-capacity transportation systems in urban areas is boosting the construction of new tunnels worldwide. Modern mechanized excavation techniques, such as 
those based on closed face TBMs with pressurized shields, usually limit tunneling-induced soil deformations and, consequently, the potential damage to structures and services, both above-ground and buried. However, problems can arise in the case of unexpected stratigraphic changes, technical malfunctioning or errors in TBM driving, hence consideration of more conservative scenarios of TBM performance is recommended for the sake of safety. In addition, traditional excavation techniques, generally associated with larger volume losses, are unavoidable in specific scenarios, e.g. for connection or platform tunnels.

In the context of tunnel-soil-building interactions, reliable predictive models are essential for optimum design. Compared to commonly employed simplified and often over-conservative approaches, interaction models should provide more accurate predictions of the ground response at different levels of volume loss, accounting explicitly for the characteristics of the buildings, including their foundation system and possible material non-linearity.

For risk assessments, the first level of investigation typically consists of a two-step uncoupled assessment of the interaction problem (Mair et al., 1996): first, the greenfield response is calculated by adopting one of the available semi-empirical expressions for ground displacements (Mair et al., 1993) and, then, the structural damage is evaluated with reference to specific greenfield deformation or displacement parameters calculated at the foundation level of the building (Burland et al., 1977; Boscardin and Cording, 1989). A more refined evaluation, needed if the category of damage resulting from this preliminary evaluation is not negligible, requires a coupled soil-structure interaction analysis in which the building can be modeled with various levels of detail, ranging from equivalent beams or solids representing the whole structure (Potts and Addenbrooke, 1997; Namazi and Mohamad, 2013; Losacco et al., 2016) to a more or less detailed description of the structural components (Son and Cording, 2005; Comodromos et al., 2014; Fargnoli et al., 2015a; Yiu et al., 2017). In most cases, studies are conducted with the aid of numerical modeling, often in three dimensions so as to accurately describe the structural layout of the building and its relative orientation with respect to the tunnel axis.

Compared to masonry buildings, relatively little attention has been devoted to the response of 
framed structures to tunneling. The peculiar response of framed buildings to excavations (Goh and Mair, 2014; Fargnoli et al., 2015b; Haji et al., 2018; Boldini et al., 2018; Fu et al., 2018) raises the need for specific damage criteria, accounting for the frame geometry (Boone, 1996; Elkayam and Klar, 2019) and for the predominant contribution of floors and walls to bending and shear stiffness respectively (Finno et al., 2005), as discussed in the next paragraph.

This paper aims at validating two different Finite Element (FE) approaches for the assessment of tunneling-induced deformation of framed structures with no or very compliant infills and the possible resulting damage on the latter, even if not explicitly modeled. Reference is made to an experimental database from recently performed centrifuge tests at the University of Nottingham, which evaluated the response of frames with varying geometry, foundation layout, stiffness and weight to the excavation of a tunnel in dry dense sand (Xu et al., 2020a,b). The performance of an advanced fully coupled FE numerical model, containing all the components of the interaction problem (i.e. the tunnel, the soil and the frame), is compared to that of a simplified two-stage FE model. Results highlight the limitations and strengths of the two numerical modeling approaches, providing useful guidance to engineering practitioners. The numerical analyses are also used to extend the scope of investigation beyond that considered experimentally, by simulating further eccentric configurations and providing further insight on the horizontal strains associated with differential displacements of buildings with separate footings.

In this paper, a review of the available methods for the assessment of deformation and damage of framed buildings is first presented, involving the estimation of a relative stiffness of the frame with respect to that of the soil. Next, the experimental campaign in sandy soil used as a comparison term is described. This is followed by the description of the numerical approaches and of the strategy adopted for parameter calibration. Finally, numerical results are compared to experimental data in terms of soil and frame displacements; the angular distortion and differential horizontal displacements, deemed the most appropriate indicator of frame deformation and expected damage of infills, if any, and their modification factors are summarized. 


\section{ASSESSMENT OF TUNNELING-INDUCED STRUCTURAL DEFORMATIONS}

The assessment of the potential tunneling-related damage of buildings requires a careful evaluation of the induced deformation field. In the well-established Critical Strain method (Boscardin and Cording, 1989), the maximum tensile strain $\varepsilon_{\max }$ in any portion of the building - i.e. either a structural partition such as a bay or panel, or any part subject to a specific deformation mode, such as sagging/hogging or predominantly shear/bending - is associated with a damage category, ranging from "negligible" to "very severe". The $\varepsilon_{\max }$ results from the composition of horizontal strains $\varepsilon_{h}$, induced by horizontal displacements, with either horizontal (bending) strains $\varepsilon_{b}$ or diagonal (shear) strains $\varepsilon_{d}$ induced by the vertical displacement field.

Traditionally, horizontal strains $\varepsilon_{h}$ are inferred from the displacements measured at the ground surface or at the foundation level, while the bending and shear strains $\varepsilon_{b}$ and $\varepsilon_{d}$ are related to either the deflection ratio $\Delta / L$ (Burland and Wroth, 1974) or the angular distortion $\beta$ (Boscardin and Cording, 1989), as defined in Figure 1. Recently, moving from Cook (1994), Ritter et al. (2020) proposed that the deformation parameters of the bay (both average curvature and shear strain) could be inferred from its top and bottom corner displacements, consistent with Xu et al. (2020a). More specifically, for framed structures with continuous foundations (e.g. rafts, grade beams transverse to the tunnel), the shear deformation $\varepsilon_{d}$ is typically dominant, as longitudinal strains due to $\varepsilon_{h}$ and $\varepsilon_{b}$ are negligible. The average shear strain level is given by the angular distortion $\beta=S-w$ of each panel or bay, as shown in Figure 1, defined as the difference between the bay slope $S$ and tilt $w$ given by the rotation of the bay edges (Boone, 1996); the angular distortion relates to the diagonal strain as $\varepsilon_{d}=\beta / 2$. For separate footings, both shear and horizontal distortions need to be considered when estimating the panel or bay deformation; in this case, the maximum strain can be approximated from a Mohr's circle for plane strain conditions by $\varepsilon_{\max }=\frac{\varepsilon_{h}+\varepsilon_{z}}{2}+\sqrt{\left(\frac{\varepsilon_{h}-\varepsilon_{z}}{2}\right)^{2}+\varepsilon_{d}^{2}}$ (Mair et al., 1996), where $\varepsilon_{h}$ and $\varepsilon_{z}$ are, respectively, the horizontal and vertical strains. Note that $\varepsilon_{z}$ may be neglected as a first approximation due to the axial action of columns restraining vertical deformations. Alternatively, vertical, horizontal, and diagonal strains may be computed directly from corner point displacements of flexible infills within bare frames (Elkayam and Klar, 2019). 
The effect of the relative soil-structure stiffness in decreasing the distortions with respect to those evaluated in greenfield conditions was first introduced by Potts and Addenbrooke (1997) in terms of modification factors of $\Delta / L$ and $\varepsilon_{h}$ for both sagging and hogging. Later, Son and Cording (2005) normalized the angular distortions of masonry building bays with respect to the differential ground slope obtained in greenfield conditions. By considering that framed configurations with axially stiff slabs/beams in the horizontal direction undergo minimal longitudinal deformations (Finno et al., 2005) and thus shear deformation is dominant, $\mathrm{Xu}$ et al. (2020a) introduced the angular distortion modification factor $M^{\beta}$ and related it to a relative soil-structure stiffness parameter $\kappa$. The latter was defined as $\kappa=E_{s} B / G A_{s}^{*}=E_{s} B L / G A_{s}$, where $E_{s}$ is the representative Young's modulus of the soil, $B$ is the building transverse length, $L$ is the length of the building in the tunnel direction, and $G A_{s}^{*}=G A_{s} / L$ is the building shear stiffness per meter run (where $G$ is the shear modulus and $A_{s}$ is the shear area contributing to shear resistance, which is only a portion of the cross-sectional area $A\left(\right.$ Cowper, 1966)). The angular distortion modification $M^{\beta}=\beta_{\max } / \overline{G S}_{\max }$ is the ratio between the maximum angular distortion of the building $\beta_{\max }$ and the maximum average greenfield slope $\overline{G S}_{\text {max }}$, both defined with respect to the building bays. When $M^{\beta}=1$, the framed building undergoes maximum shear deformations equal to the largest greenfield slope. It should be self-evident that the reliable application of this approach, or other similar methods, requires the implementation of rational procedures to estimate representative values of soil and structure stiffness.

Finally, a modification factor for compressive and tensile horizontal strains between separate footings, caused by horizontal ground movements, is also considered. This is defined as $M^{\varepsilon_{h}}=\varepsilon_{h, \text { max }}^{\text {bld }} / \varepsilon_{h, \text { max }}^{g f}$, where $\varepsilon_{h, \text { max }}^{\text {bld }}$ is the maximum horizontal strain at the building foundation and $\varepsilon_{h, \max }^{g f}$ is the largest average strain inferred from the greenfield displacements at the footing locations (Dimmock and Mair, 2008). The relative structure-soil stiffness is inferred from an analysis of the response of a single portal, with one story and a single bay, to a differential horizontal displacement (Goh and Mair, 2014). This approach provides the dimensionless factor $\alpha_{f}^{*}=1 /(E s L) \times 3 K_{b} K_{c} /\left(h_{\text {story }}^{2}\left(2 K_{b}+3 K_{c}\right)\right)$, where $K_{c}=E I_{c} / h_{\text {story }}$ and $K_{b}=E I_{c} / b_{\text {bay }}, E I_{c}$ and 
$E I_{b}$ are the bending stiffness of the column and the first-floor slabs, $h_{\text {story }}$ is the column height, and $b_{\text {bay }}$ is the bay length.

\section{REPRESENTATIVE SOIL STIFFNESS}

To evaluate a representative value of Young's modulus for the soil $E_{s}$, Mair (2013) suggested that the tunneling-induced level of shear strain should be considered in combination with an appropriate soil stiffness degradation curve. In this paper, the approach of Marshall et al. (2010) and Farrell (2010) is adopted, considering ground stresses and strains at mid-depth $z_{t} / 2$, where $z_{t}$ is the depth to the tunnel axis.

Firstly, the soil stiffness degradation curve is acquired (i.e. the relationship between the shear strain level $\gamma_{s}$ and the relative reduction of secant shear modulus $G_{s}$ with respect to the initial "small-strain" modulus $G_{0}$ ). The small-strain stiffness should be adjusted to account for relative density and mean effective stress, e.g. using, for example, the expressions proposed by Lehane and Cosgrove (2000). Secondly, the average shear strain level $\gamma_{s}$ experienced by the soil during tunneling in greenfield conditions is evaluated for a given tunnel volume loss $V_{l, t}$ (i.e. the relative change in tunnel cross-sectional area). In order to obtain $\gamma_{s}$, the shear strain distribution at $z_{t} / 2$ is averaged between $\pm 2.5 i$, where $i$ is the offset from the tunnel centerline to the settlement trough inflection point. Then, by assuming a value of Poisson's ratio for the soil $v_{s}$, the representative value of the soil stiffness $E_{s}$ is computed for any $V_{l, t}$.

\section{EQUIVALENT FRAME STIFFNESS}

Equivalent Timoshenko and laminated beams can be employed as a simplified structural model, with the advantage of allowing separate control of the bending $(E I)$ and shear $\left(G A_{S}\right)$ contribution (Finno et al., 2005; Pickhaver et al., 2010; Franza et al., 2020) to the overall building stiffness. This approach can be contrasted with that of the pure bending stiffness $E I_{E B}$ based on the Euler-Bernoulli beam theory (Franzius et al., 2006; Goh and Mair, 2014; Haji et al., 2018). The equivalent bending and shear stiffness are typically estimated by analytical methods (Franzius et al., 2006; Finno et al., 2005; Pickhaver et al., 2010) and loading tests, carried out either experimentally or numerically (Son and Cording, 2005; Xu et al., 2020a; Losacco et al., 2014, 2016). 
In this paper, the equivalent bending (EI) stiffness is analytically obtained from the parallel axis theorem, using the cross-sectional areas of the floor slabs. Next, the shear stiffness $G A_{s}$ is estimated from a loading test of a simply supported framed structure subjected to a concentrated load, similar to Goh and Mair (2014). For the Timoshenko beam theory, the deflection-to-force ratio $\delta / P$ can be expressed as:

$$
\frac{\delta}{P}=b \frac{B^{3}}{\left(\frac{E I}{1+a F}\right)}
$$

where $P$ is the total applied force, $B$ is the beam length, and $F=(E I) /\left(B^{2} G A_{s}\right)$. The adopted coefficients $a=12$ and $b=1 / 48$ depend on the selected boundary conditions. It follows from Equation (1) that the shear stiffness $G A_{s}$ is given by:

$$
\frac{1}{G A_{S}}=\frac{B^{2}}{a E I}\left(\frac{\delta}{P} \frac{E I}{b B^{3}}-1\right)
$$

when using $\delta / P$ analytical estimated from a loading test and bending stiffness $E I$.

This single equation approach based on Equation (2) and the use of the parallel axis theorem was validated against the shear stiffness values obtained from multiple experimental loading tests carried out by Xu et al. (2020a,b). The single equation approach predicted slightly smaller (within $10 \%$ ) stiffness values with respect to the experiments. Therefore, using the parallel axis theorem to calculate the equivalent $E I$ with Equation (2) is a reasonable approximation.

\section{DESCRIPTION OF CENTRIFUGE TESTS}

In this paper, centrifuge tests of tunneling beneath a framed building are considered (Xu et al., 2020a,b). At prototype scale, the tunnel has diameter $D_{t}=6.1 \mathrm{~m}$ and a cover depth $C=8 \mathrm{~m}$ $\left(C / D_{t}=1.3\right)$. For the frames, Table 1 provides details of the considered configurations and Figure 2 shows the layout with an illustration of relevant parameters. In this paper, frames are labeled following Xu et al. (2020b) as FxtybzL or FxtybzS: $\mathrm{x}$ is the number of stories, $\mathrm{y}$ the thickness of structural elements at centrifuge model scale, $\mathrm{z}$ is the number of bays, while $\mathrm{L}$ and $\mathrm{S}$ stands for long and short building, respectively. Tunnel volume losses up to $3 \%$ were considered, 
although most of the numerical results are reported for $V_{l, t}=1$ and $2 \%$.

The experiments were performed at 68 times normal gravity $(68 \mathrm{~g})$ and used a plane-strain set-up. Within the strongbox, a flexible cylindrical membrane filled with water simulates the tunnel; excavation is reproduced by extracting a measured volume of water from the membrane, thus controlling the tunnel volume loss. A dry fine-grained silica sand, known as Leighton Buzzard Fraction E, was used for the soil; this material is characterized by minimum and maximum void ratios of 0.65 and 1.01, respectively. All considered experiments were performed with a soil relative density $I_{d}=90 \%$, to which the numerical study exclusively refers. Triaxial tests on this material were carried out by Zhao (2008) and Visone (2008), data from which were used to evaluate soil representative stiffness and calibrate the advanced numerical models, respectively (details provided in a subsequent section).

Model frames were made of aluminum, consisting of vertical walls and horizontal slabs that extended $258 \mathrm{~mm}$ in the longitudinal tunnel direction, leaving a $1 \mathrm{~mm}$ gap between the frame and the front/back strongbox walls. To achieve a rigid wall-slab connection, adjoining model frame parts were welded together along approximately $60 \%$ of the connected lengths (in the tunnel direction). A layer of sand was glued to the base of the bottom slab to provide a rough soil-raft foundation interface. After centrifuge testing with the frame on raft foundation, the same model was modified to create the separate footings configuration (by machining out portions of the bottom slab). Note that the welding process did result in some asymmetric response of the frame to loading, which will have affected horizontal footing displacements in the centrifuge tests; this was discussed in detail in Xu et al. (2020b).

An experimental parametric study of the tunnel-frame interaction problem was performed by varying the geometry, stiffness, weight, foundation type, and eccentricity $e$ of the structure with respect to the tunnel centerline. As detailed in Table 1: the number of stories was either 2 or 5; the number of bays was either 3 or 6 ; the bay length was either 5.2 or $10.4 \mathrm{~m}$ (prototype scale), the latter for the frame with 3 bays only; the thickness $t$ of the structural elements was either 0.32 or $0.22 \mathrm{~m}$ (prototype scale); the eccentricity to frame width ratio, $e / B$, was either zero ("centered" 
cases) or 0.5 ("eccentric" cases); the weight of the frame was either its own self-weight (indicated as SW) or double the self-weight (indicated as $2 \mathrm{SW}$ ), achieved by adding masses to the top of the frame in a way that did not alter the structural stiffness. A total of 12 tests was performed with frames on raft foundations, whereas 6 tests were conducted for frames on separate footings, where the footing width $b_{\text {foot }}=0.8 \mathrm{~m}$ (prototype scale).

\section{DETAILS OF NUMERICAL MODELING}

In this section, the two FE approaches adopted for the numerical investigation are described. The advanced numerical model requires detailed information on soil behavior and structural characteristics, along with associated requirements of computational and post-processing costs. On the other hand, the two-stage model is suitable for quick preliminary estimates and sensitivity studies because of the limited number of required inputs as well as its negligible execution time.

The simulations with the advanced model were carried out more or less simultaneously with the experimental campaign in the centrifuge. The outcomes of the experiments were not known and only the results of the loading tests on the frame were available at the time, hence the analyses can be considered as Class B predictions (Lambe, 1973). The fully coupled modeling technique was used to simulate all centrifuge tests in Table 1, alternatively see Table S1 of "Supplemental Materials". After verifying the accuracy of the predictions, the same technique was then employed to explore the impact of tunnel-building eccentricity on the deformations of the frame. Seven additional simulations were performed: frames F2t3b3L and F2t3b6L founded on both footings and rafts for $e / B=0.5$ and SW/2SW weight conditions, except for the F2t3b3L 2SW case on footings, which did not converge.

The two-stage approach was employed to perform a Class A prediction (i.e. before the experiment was carried out, but with available experimental information on greenfield tunneling and its effects on buildings in similar conditions) of the frame F2t3b6L on a raft foundation. Subsequently, the full set of analyses was performed again after the centrifuge tests were completed (class $\mathrm{C}$ predictions), using the experimental greenfield data as an input. 


\section{Advanced model}

The advanced numerical model was set up using the commercial FE software Abaqus (version 6.14). Given the problem geometry and boundary conditions, plane strain analyses were carried out. A sample FE mesh, for case F2t3b6L with separate footings, is shown by Figure S1 in "Supplemental Materials". First-order, 4-noded plane strain elements with full integration were adopted for the soil, whereas second-order 8-noded elements with reduced integration were used for the frame. Conventional boundary conditions were applied: horizontal displacements prevented along the sides; both vertical and horizontal displacements prevented along the base.

Regarding the simulation steps, a gravitational lithostatic stress field was initially applied to the soil assuming a coefficient of earth pressure at rest $K_{0}=0.5$. The self-weight of the frame was then slowly activated in order to achieve equilibrium. A no-penetration, Coulomb-friction contact law was enforced between the ground surface and the foundation, assuming a coefficient of friction $\tan \left(\phi_{c s}^{\prime}\right)$, with $\phi_{c s}^{\prime}=32^{\circ}$ as the critical state friction angle of the soil. Subsequently, tunnel excavation was simulated in a simplified fashion by incrementally applying a prescribed displacement field at the tunnel boundary after removing the soil elements (Cheng et al., 2007). This technique has proven capable of achieving a realistic greenfield subsidence profile at the ground surface (Rampello et al., 2012; Amorosi et al., 2014). The prescribed tunnel boundary displacements, the magnitude of which depend on the target $V_{l, t}$, were defined to obtain a homothetic contraction of the tunnel cross-section centered on the tunnel invert.

The advanced constitutive model SANISAND (Dafalias and Manzari, 2004) was adopted to simulate the soil response from very small to medium strain levels $\left(V_{l, t}\right.$ as large as $3 \%$ was generally reached in the numerical analyses). The calibration of material parameters, reported in Table S2 of "Supplemental Materials", was based on a mixed strategy, considering experimental data of the Fraction E sand used in the centrifuge tests, for similar relative densities. In particular, starting from the values reported in Giardina et al. (2020), a calibration process was carried out with reference to the laboratory tests performed by Visone (2008), consisting of drained and undrained triaxial compression and extension tests as well as resonant column and torsional shear tests. The final 
set of values listed in "Supplemental Materials" was obtained by performing a further parametric study on two specific constants, i.e. $h_{0}$, controlling the plastic modulus, and $A_{0}$, governing the dilatancy law, aimed at reproducing the greenfield tunneling-induced displacements presented in Farrell et al. (2014). This approach, i.e. calibrating numerical parameters based on the simulations of the greenfield boundary value problem, is believed to be more robust than only using results from element-scale laboratory tests. Indeed, Figure S3 in "Supplemental Materials" demonstrate an excellent match between numerical and experimental results in terms of the relationship between tunnel volume loss $V_{l, t}$ and ground surface volume loss $V_{l, s}$ (where $V_{l, s}$ is the area of the surface settlement trough divided by the nominal area of the tunnel cross-section).

For the frame, a simple linear elastic constitutive law was adopted with Young's modulus $E=53.8 \mathrm{GPa}$, Poisson's ratio $v=0.334$ and unit weight $\gamma=27 \mathrm{kN} / \mathrm{m}^{3}$. The reduced value of $E$ used for the aluminum frame, instead of the standard $70 \mathrm{GPa}$, was selected to account for the partial welding of the frame components (described earlier); this value of $E$ was found by simulating load-deflection tests carried out on the frames (Xu et al., 2020a).

\section{Simplified model}

The performance of the advanced model was compared to that of the simplified elasticity-based two-stage FE model called Analysis of Structural Response to Excavation (ASRE) (Franza and DeJong, 2019; Franza et al., 2020). The mechanical components of the model are described as follows (sketched in Figure S2 "Supplemental Materials"). The structure, incorporating both the superstructure and foundation, is modeled as a frame consisting of Euler-Bernoulli beam elements with geometry and material properties of the prototype building; the self-weight was simulated as line loads applied along the beam axes. The structure is founded on coupled elastic springs simulating the ground as an elastic half-space of Young's modulus $E_{s}$ and Poisson's ratio $v_{s}$. The effects of tunnel excavation are simulated through a set of equivalent forces applied to the springs that reproduce the ground movements observed in greenfield conditions. In other words, in elasticity-based two-stage methods, (1) greenfield movements are firstly estimated and then (2) the soil-structure system is solved for the forces associated with these greenfield movements. It follows 
that two-stage methods are approximated in case of soil non linearity, while they provide an exact solution for linear elastic soil-structure systems.

Two types of simplified analyses were conducted: linear elastic, labeled 'EL', and elastoplastic 'EP'. For the EP analyses, plastic sliders are located at the soil-foundation interface such that horizontal and vertical tensile forces are limited, capturing slipping and gap formation mechanisms. In the EP analyses, the self-weight of the structure needs to be applied prior to simulating the tunnel excavation. In the elastic EL analyses, a perfect soil-foundation compatibility condition was assumed by deactivating the sliders.

Numerical simulations were carried out before (i.e class A predictions (Lambe, 1973)) and after (i.e. class $\mathrm{C}$ predictions) the centrifuge tests. When selecting the plane frame model parameters, $E=70 \mathrm{GPa}$ and $54 \mathrm{GPa}$ were assumed for the Class A and Class $\mathrm{C}$ predictions, respectively, because the influence of incomplete welding was not accounted for prior to the experiments. Also, the length of the structure in the tunnel direction $L$ was set equal to $10 \mathrm{~m}$. For the ground, a representative Young's modulus of $E_{s}=45 \mathrm{MPa}$ and a Poisson's ratio of $v_{s}=0.3$ were assumed for the elastic half-space. For the plastic sliders, a friction coefficient corresponding to that of the soil at critical state (i.e. $32^{\circ}$ ) and zero tensile strength were used. Centrifuge results of greenfield tunneling reported by Farrell et al. (2014) and Xu et al. (2020a) were used to define the inputs for Class A and C simulations, respectively.

\section{RESULTS OF THE ADVANCED MODEL}

\section{Comparison between numerical and centrifuge results: ground surface displacements}

Numerical results and centrifuge data are compared in this section in terms of tunneling-induced settlements $U_{z}$ and horizontal displacements $U_{x}$ at the ground surface (these latter shown in "Supplemental Materials" for the raft foundation case due to their negligible importance for this type of foundation). Figures 3 and S4 show the settlements and horizontal displacements, respectively, for the raft foundation cases, while Figures 4 and 5 relate to separate footings. The subplots are arranged from top to bottom with increasing relative structural stiffness. All the displayed results 
refer to a tunnel volume loss of $1 \%$; for the sake of completeness, corresponding plots are provided in "Supplemental Materials" for a tunnel volume loss of 2\% (Figures S5-S8).

The comparison in terms of settlements is generally good for frames founded on rafts (Figure 3), but less good for frames on separate footings (Figure 4). The centrifuge results indicate a gap between the underlying soil and the raft foundation for the three stiffer frames with nominal selfweight SW (Figure 3(c-e), SW case). Numerically, however, a gap was only detected for the raftfounded SW frames in test F2t5b6L for $V_{l, t}=1 \%$ (Fig. 6(i)) and in both tests F2t5b6L and F5t5b6L for $V_{l, t}=2 \%$ (Fig. S5(i,j)). For frames on separate footings, a gap was not observed in the centrifuge nor in numerical results, even at $V_{1, \mathrm{t}}=2 \%$ (see "Supplemental Materials"), though the numerical simulations tend to underestimate centrifuge test footing settlements. The influence of structural stiffness and weight on settlements is well captured by the numerical model for the raft foundation cases. Here, irrespective of the tunnel volume loss, the larger the frame stiffness, the smaller the maximum and differential settlements, which are also always smaller than in the greenfield case, at least for the long frame configurations. In the experiments, the additional applied weight (i.e. 2SW) was capable of remarkably altering the settlement distribution at the foundation level, particularly in the central portion of the structure. This behavior is reproduced only marginally, mainly for the stiffer frames, by the advanced FE simulations. Also, for frames with separate footings (Figure 4), the computed FE settlement distribution appears only slightly affected by the frame stiffness at the global level, the response differing from that of the greenfield curve only locally, where the footings are located. The centrifuge data show more marked local settlements, especially for the eccentric case (Figures 4(b) and S7(b)) for which much larger maximum settlements were recorded. Several possible reasons could explain such behavior, ranging from experimental difficulties in guaranteeing a uniform soil density in the centrifuge, or the use of a soil mesh close to the footings that was not sufficiently fine and therefore incapable of describing the localized displacement gradients. For both the experiments and the simulations, the settlements under the footings appear relatively insensitive to the applied self-weight.

Horizontal displacements predicted at the base of the raft foundation (see "Supplemental Ma- 
terials") are negligible for all investigated cases, similar to the results from the centrifuge tests. As such, most of the numerical simulations, similar to the experiments, are characterized by sliding at the soil-structure interface, progressively reducing in extension and intensity to zero as the stiffness and weight of the frame increases. A completely different pattern was found for the separate footing cases at $V_{l, t}=1 \%$ (Figure 5): sliding at the soil-structure interface was never observed in the centrifuge nor predicted in the numerical analyses. Horizontal displacements are moderately lower than those obtained in greenfield conditions, showing local reductions directly beneath the footings. Only the eccentric case (Figures 5(b) and (e)) provides deformations from both centrifuge data and numerical simulations that are slightly larger than in greenfield conditions, as the footings farther from the tunnel centerline were possibly dragged towards the nearer footings by the overall frame movement. The increase of volume loss, considered in "Supplemental Materials", does not modify these observations, though a modest effect of structural weight can be detected and some slight slippage occurs under the central footings for the stiffer cases with $e / B=0$ and nominal applied self-weight SW both in the experiments and in the simulations. Note that differential horizontal movements between footings are possible only when no ground floor slab or grade beam is present and infills are flexible.

\section{Deformation parameters}

A concise representation of numerical results and their comparison with centrifuge data is provided in terms of maximum angular distortion $\beta_{\max }$ ( $\operatorname{sign}$ was not considered) in Figures 6 and 7 for rafts and separate footings, respectively, for $V_{l, t}$ up to $3 \%$.

The overall trend outlined by the centrifuge results is well captured by the numerical predictions, especially for the analyses of frames founded on raft foundations, the $\beta_{\max }$ values being generally slightly overestimated in the numerical analyses. The $\beta_{\max }$ increases with $V_{l, t}$, with values lower than $0.3 \%$ for both rafts and separate footings. Eccentricity of the frame has a significant beneficial effect in limiting the structural distortion in comparison to the central configuration, while a detrimental influence can be observed for the building weight (i.e. 2SW analyses are always characterized by larger values of $\beta_{\max }$ ). 


\section{RESULTS OF THE SIMPLIFIED MODEL}

The performance of the simplified ASRE model for both linear elastic EL (perfect soil-foundation compatibility) and elastoplastic EP (with active sliders) conditions is compared with the centrifuge data of the F2t3b6L frame founded on the raft (for brevity, only this case is discussed here). Figure 8 shows tunneling-induced settlements of the foundations and angular distortions of bays for central frames at $V_{l, t}=2 \%$. Horizontal raft displacements are not reported since they are nearly zero for the central frame cases, as previously discussed for the advanced modeling results.

First, the Class A predictions of the frame model in Figures 8(a) and (c) are discussed. As noted earlier, these analyses were performed prior to collecting the centrifuge data to evaluate the accuracy of the two-stage model. In this ASRE analysis, despite the use of a greenfield input from Farrell et al. (2014) with slightly greater movements than Xu et al. (2020a) (compare solid and dashed lines in Figure $8(\mathrm{a}, \mathrm{c}))$, the maximum building settlement was predicted well by the elastoplastic EP analysis, due to its capability of considering gap formation, which is not allowed in the elastic EL case. The building settlement shape is also reproduced reasonably well by both the EL and EP analyses. This is confirmed by the comparison of the bay $\beta$ values along the building length, with ASRE results providing a satisfactory estimate of experimental outcomes, and only a marginal difference between EL and EP results.

Class C estimates, displayed in Figures 8(b) and (d), are considered to evaluate the implications of using different greenfield inputs (the Class $\mathrm{C}$ greenfield input is directly applicable to the tunnelframe interaction centrifuge results presented here). The difference in the foundation settlements between the EP and EL solutions is minimal when adopting the greenfield movements from Xu et al. (2020a), indicating limited slider displacements for the EP case. The comparison between ASRE and experimental results in terms of maximum building settlement and $\beta$ is also acceptable, as for the advanced FE model results.

\section{MODIFICATION FACTORS}

To synthesize data in design charts for use within preliminary risk assessments, this section provides angular distortion and horizontal strains at the foundation level using modification factors and 
recently proposed relative stiffness terms. To further populate the dataset of eccentric structures with relatively high frame flexibility, additional numerical analyses were run with the advanced FE model using an enlarged mesh, required to accommodate the full length of the long eccentric frames (e.g. cases F2t3b3L and F2t3b6L for $e / B=0.5$ ). Furthermore, the ASRE model was used to simulate all frames in Table 1 under central and eccentric conditions $(e / B=0 ; 0.5)$ using the elastoplastic EP analysis method. Also note that results computed at $V_{l, t}=1$ and $2 \%$ are considered for the advanced FE model and centrifuge results, whereas only $V_{l, t}=2 \%$ is selected for ASRE considering that, for the simplified method, there is a limited effects of $V_{l, t}$.

Modification factors for the angular distortion, $M^{\beta}$, derived from all the advanced and ASRE numerical analyses are plotted in Figures 9 and 10 against the relative soil-structure stiffness parameter $\kappa$ for the raft and separate footings cases, respectively. Values of $\beta$ refer to panels confined by two slabs and two columns, while horizontal strains due to differential horizontal displacements of separate footing are not accounted for. These data are compared on the same charts with the corresponding centrifuge test values and with the empirical upper and lower envelopes (based on centrifuge test data) proposed by Xu et al. (2020a,b).

Figure 9 indicates that, for raft foundations, all the numerical results fit relatively well within the empirical envelopes for both the centered and eccentric frames. For each examined case with $e / B=0$, both FE predictions yield a somewhat larger distortion for a given maximum ground slope, the difference between experimental and numerical values being larger for the more flexible cases. In contrast, for $e / B=0.5$, Abaqus numerical data points tend to concentrate near the lower envelope for the eccentric frames on raft foundations. Also, the ASRE simulations indicate a rate of variation of $M^{\beta}$ against relative stiffness $\kappa$ that is lower than the empirical envelopes.

As seen in Figure 10, there is agreement between experimental and numerical factors for frames with separate footings, with the numerical data points tending to be located close to the upper envelope for $e / B=0$. Similar to the case of the raft foundation, the agreement between ASRE and advanced results are less good for the eccentric frames on separate footings than they are for the centered frames. This may be partly due to the way that eccentric frames affect the tunneling- 
induced arching mechanism, which is not considered by the elastic continuum used in the ASRE model.

Overall, numerical results confirm that the envelopes proposed by Xu et al. (2020a,b) are reasonable for a wider range of scenarios. Also, Figures 9 and 10 allow for a direct comparison between advanced and ASRE predictions in terms of normalized angular distortions, indicating a good agreement except for relatively flexible eccentric frames. This difference for flexible eccentric frames occurred as a result of the building weight effect, which slightly increases tunneling-induced settlements, a mechanism not considered by ASRE.

To illustrate the influence of bay relative stiffness and building eccentricity on horizontal deformations, numerical results of the modification factor for horizontal strains $M^{\varepsilon_{h}}$ obtained from advanced and simplified models are compared in Figures 11. In this figure, values of $M^{\varepsilon_{h}}$ were computed from the maximum differential horizontal displacements of greenfield and building displacement profiles at the footing locations. Centrifuge results are not considered because of the previously mentioned effects of welding on the horizontal displacements of the footings (Xu et al., 2020b). Interestingly, both models predicted horizontal modification factors $M^{\varepsilon_{h}}$ lower than unity in both compression and tension (i.e. a semi-flexible behavior), with no clear trends associated with the change in eccentricity $e / B$. For a given frame and location, the reduction in the building self-weight slightly reduced the horizontal deformations in the advanced model for all cases, while its impact on ASRE results is significant in compression for the eccentric two story frames that are relatively stiff in shear (namely, F2t3b3S and F2t5b6L). More importantly, in most cases the level of predicted normalized horizontal deformation in the advanced approach is notably lower than that resulting from the ASRE predictions, likely due to the former model accounting for the ground stiffness degradation related to the footing restraint action in the horizontal direction, as displayed in Figure 5. Finally, considering the full parametric study conducted with ASRE, the decrease in $M^{\varepsilon_{h}}$ with the relative stiffness $\alpha_{f}^{*}$ is notable only when the cross-sectional thickness is increased, resulting in values of $\alpha_{f}^{*}$ being greater by approximately one order of magnitude. 


\section{CONCLUSIONS}

The paper describes a numerical study intended to verify the capabilities of numerical approaches, characterized by different levels of complexity, in reproducing the response of bare frame buildings to tunneling in sand, as observed during centrifuge tests considering both raft foundations and separate footings. Numerical modeling was also used to expand the available centrifuge dataset by analyzing additional eccentric cases.

The numerical models, all based on the finite element method, were established with two aims: on one side, executing advanced simulations of the interaction problem by explicitly including the tunnel, the soil and the frame with its foundation; on the other side, developing more simplified tools for the engineering practice, without the need of running time-consuming analyses and of adopting advanced constitutive models. The latter are two-stage models in which the frame is modeled through a frame consisting of beams, the soil is substituted by coupled springs with optional plastic sliders at the soil-structure interface, while tunneling is input in terms of greenfield movements. In both the advanced and simplified FE models, the behavior at the soil-building interface can be specifically accounted for by limiting the allowable tangential stress and by setting the tensile strength to zero.

Both the discussed numerical approaches were able to capture settlements and angular distortions of the frame bays for both rafts and separate footings. The accuracy of the advanced numerical model can be attributed to various factors: a proper, even if simplified, simulation of tunnel excavation; the use of an advanced constitutive law for the sand, the capability of correctly reproducing the tunneling-induced subsidence throughout a relatively large range of volume loss values, over $2 \%$; and the use of contact laws to allow for the occurrence of sliding and the formation of a gap below the frame foundation, as observed experimentally. Notably, it was demonstrated that good and quick estimates of settlements and building distortions can be achieved for framed structures with the simplified ASRE model; these can subsequently be refined when more representative greenfield data become available.

Approximated approaches for the estimation of both bending and shear stiffness were presented 
and validated. The whole set of numerical results was interpreted in terms of modification factors for both angular distortion and horizontal strain in relation to relative soil-building stiffness. These angular distortion results agreed well with previously proposed empirical envelopes (Xu et al., 2020a,b), defined on the basis of centrifuge outcomes, that can bound, with reasonable success, the range of predicted angular distortions, considering the impact of foundation type (i.e. raft or separate footings) and relative soil-structure stiffness. Additionally, indications were given on expected ranges of horizontal strains caused by the differential horizontal displacements between separate footings. Numerical results confirmed that shear deformations play an important role for all considered buildings, whereas only frames on separate footings are sensitive to horizontal ground movements.

The envelopes of modification factors may be of use for a preliminary assessment of the reduction of bay angular distortion in comparison to the greenfield case. Alternatively, the simplified numerical approach represents a viable tool for a prompt preliminary assessment, which also accounts for many important structural characteristics that are not considered in the proposed envelopes (e.g. bay length-to-height ratio, different stiffness of columns and floors).

In this paper no explicit structural model of the infills was considered, which may have a significant impact on the response of the frame due to their stiffening effect. Therefore, the obtained results and current assessment procedures are deemed conservative if applied within the context of tunneling beneath infilled frames. Future works will provide further insights into both the stiffening action as well as the deformations of infills of framed buildings.

\section{DATA AVAILABILITY}

Data and models are available from the authors on request.

\section{ACKNOWLEDGEMENTS}

This project has received funding from the European Union's Horizon 2020 research and innovation programme under the Marie Sklodowska-Curie grant agreement No 793715. The financial support provided by the China Scholarship Council (CSC) and the University of Nottingham, UK, is also recognized. 


\section{REFERENCES}

Amorosi, A., Boldini, D., De Felice, G., Malena, M., and Sebastianelli, M. (2014). "Tunnellinginduced deformation and damage on historical masonry structures." Géotechnique, 64(2), 118-130. doi:10.1680/geot.13.P.032.

Boldini, D., Losacco, N., Bertolin, S., and Amorosi, A. (2018). "Finite Element modelling of tunnelling-induced displacements on framed structures." Tunn. Undergr. Sp. Technol., 80(Oct), 222-231. doi:10.1016/j.tust.2018.06.019.

Boone, S. J. (1996). "Ground-Movement-Related Building Damage.” J. Geotech. Eng., 122(11), 886-896. doi:10.1061/(ASCE)0733-9410(1996)122:11(886).

Boscardin, M. D. and Cording, E. J. (1989). "Building response to excavation-induced settlement." $J$. Geotech. Eng., 115(1), 1-21. doi:10.1061/(ASCE)0733-9410(1989)115:1(1).

Burland, J. B., Broms, B. B., and De Mello, V. F. B. (1977). "Behaviour of foundations and structures." Proc. 9th Int. Conf. Soil Mech. Found. Eng., Vol. 2, Tokyo, 495-546.

Burland, J. B. and Wroth, C. P. (1974). "Settlement of buildings and associated damage." Proc. Conf. Settl. Struct., London, UK, Pentech Press, 611-654.

Cheng, C. Y., Dasari, G. R., Chow, Y. K., and Leung, C. F. (2007). "Finite element analysis of tunnelsoil-pile interaction using displacement controlled model." Tunn. Undergr. Sp. Technol., 22(4), 450-466. doi:10.1016/j.tust.2006.08.002.

Comodromos, E. M., Papadopoulou, M. C., and Konstantinidis, G. K. (2014). "Numerical Assessment of Subsidence and Adjacent Building Movements Induced by TBM-EPB Tunneling." J. Geotech. Geoenvironmental Eng., 140(11), 04014061. doi:10.1061/(ASCE)GT.1943-5606.0001166.

Cook, D. (1994). "Studies of settlement and crack damage in old and new facades." Proc., 3rd Int. Mason. Conf. London, Engl., Vol. 6, 203-211.

Cowper, G. R. (1966). “The Shear Coefficient in Timoshenko's Beam Theory.” J. Appl. Mech., 33(2), 335-340. doi:10.1115/1.3625046.

Dafalias, Y. F. and Manzari, M. T. (2004). "Simple Plasticity Sand Model Accounting for Fabric Change Effects.” J. Eng. Mech., 130(6), 622-634. doi:10.1061/(ASCE)0733-9399(2004)130:6(622).

Dimmock, P. S. and Mair, R. J. (2008). "Effect of building stiffness on tunnelling-induced ground movement." Tunn. Undergr. Sp. Technol., 23(4), 438-450. doi:10.1016/j.tust.2007.08.001.

Elkayam, I. and Klar, A. (2019). "Nonlinear elasto-plastic formulation for tunneling effects on superstructures." Can. Geotech. J., 56(7), 956-969. doi:10.1139/cgj-2018-0021.

Fargnoli, V., Boldini, D., and Amorosi, A. (2015a). "Twin tunnel excavation in coarse grained soils: Observations and numerical back-predictions under free field conditions and in presence of a surface structure." Tunn. Undergr. Sp. Technol., 49, 454-469. doi:10.1016/j.tust.2015.06.003.

Fargnoli, V., Gragnano, C. G., Boldini, D., and Amorosi, A. (2015b). "3D numerical modelling of soil-structure interaction during EPB tunnelling." Géotechnique, 65(1), 23-37. doi:10.1680/geot.14.P.091.

Farrell, R. (2010). "Tunnelling in sands and the response of buildings." Ph.D. Thesis, Cambridge Univ.

Farrell, R., Mair, R., Sciotti, A., and Pigorini, A. (2014). "Building response to tunnelling." Soils Found., 54(3), 269-279. doi:10.1016/j.sandf.2014.04.003.

Finno, R. J., Voss, F. T., Rossow, E., and Blackburn, J. T. (2005). "Evaluating Damage Potential in Buildings Affected by Excavations." J. Geotech. Geoenvironmental Eng., 131(10), 1199-1210. doi:10.1061/(ASCE)1090-0241(2005)131:10(1199). 
Franza, A., Acikgoz, S., and DeJong, M. J. (2020). "Timoshenko beam models for the coupled analysis of building response to tunnelling." Tunneling Undergr. Constr., 96(February), 103160. doi:10.1016/j.tust.2019.103160.

Franza, A. and DeJong, M. J. (2019). "Elastoplastic solutions to predict tunneling-induced load redistribution and deformation of surface structures." J. Geotech. Geoenvironmental Eng., 145(4), 04019007. doi:10.1061/(ASCE)GT.1943-5606.0002021.

Franzius, J. N., Potts, D. M., and Burland, J. B. (2006). “The response of surface structures to tunnel construction." Proc. ICE - Geotech. Eng., 159(1), 3-17. doi:10.1680/geng.2006.159.1.3.

Fu, J., Yu, Z., Wang, S., and Yang, J. (2018). "Numerical analysis of framed building response to tunnelling induced ground movements.” Eng. Struct., 158(Mar), 43-66. doi:10.1016/j.engstruct.2017.11.039.

Giardina, G., Losacco, N., DeJong, M. J., Viggiani, G. M. B., and Mair, R. J. (2020). "Effect of soil models on the prediction of tunnelling-induced deformations of structures." Proc. Inst. Civ. Eng. - Geotech. Eng., 173(5), 379-397. doi:10.1680/jgeen.18.00127.

Goh, K. H. and Mair, R. J. (2014). "Response of framed buildings to excavation-induced movements." Soils Found., 54(3), 250-268. doi:10.1016/j.sandf.2014.04.002.

Haji, T. K., Marshall, A. M., and Tizani, W. (2018). "A cantilever approach to estimate bending stiffness of buildings affected by tunnelling." Tunn. Undergr. Sp. Technol., 71(Jan), 47-61. doi:10.1016/j.tust.2017.08.005.

Lambe, T. W. (1973). "Predictions in soil engineering." Géotechnique, 23(2), 151-202. doi:10.1680/geot.1973.23.2.151.

Lehane, B. and Cosgrove, E. (2000). "Applying triaxial compression stiffness data to settlement prediction of shallow foundations on cohesionless soil." Proc. ICE - Geotech. Eng., 143(4), 191-200. doi:10.1680/geng.2000.143.4.191.

Losacco, N., Burghignoli, A., and Callisto, L. (2014). "Uncoupled evaluation of the structural damage induced by tunnelling." Géotechnique, 64(8), 646-656. doi:10.1680/geot.13.P.213.

Losacco, N., Callisto, L., and Burghignoli, A. (2016). "Soil-structure interaction due to tunnelling in soft ground, an equivalent solid approach." Struct. Anal. Hist. Constr., K. Van Balen and E. Verstrynge, eds., number 2010, CRC Press, 495-501. doi:10.1201/9781315616995-74.

Mair, R. (2013). "Tunnelling and deep excavations: ground movements and their effects." Proc. 15th Eur. Conf. Soil Mech. Geotech. Eng. - Geotech. Hard Soils - Weak Rocks (Part 4), A. Anagnostopoulos, M. Pachakis, and C. Tsatsanifos, eds., Amsterdam, the Netherlands, IOS Press, 39 - 70.

Mair, R. J., Taylor, R. N., and Bracegirdle, A. (1993). "Subsurface settlement profiles above tunnels in clay." Géotechnique, 43(2), 315-320. doi:10.1680/geot.1993.43.2.315.

Mair, R. J., Taylor, R. N., and Burland, J. B. (1996). "Prediction of ground movements and assessment of risk of building damage due to bored tunnelling." Proc. Int. Symp. Geotech. Asp. Undergr. Constr. Soft Gr., R. J. Mair and R. N. Taylor, eds., London, United Kingdom, Balkema, Rotterdam, 713-718.

Marshall, A. M., Klar, A., and Mair, R. J. (2010). "Tunneling beneath buried pipes: View of soil strain and its effect on pipeline behavior." J. Geotech. Geoenvironmental Eng., 136(12), 1664-1672. doi:10.1061/(ASCE)GT.1943-5606.0000390.

Namazi, E. and Mohamad, H. (2013). "Assessment of Building Damage Induced by ThreeDimensional Ground Movements." J. Geotech. Geoenvironmental Eng., 139(4), 608-618. doi:10.1061/(ASCE)GT.1943-5606.0000822.

Pickhaver, J., Burd, H., and Houlsby, G. (2010). "An equivalent beam method to model masonry buildings in 3D finite element analysis.” Comput. Struct., 88(19), 1049-1063. doi:10.1016/j.compstruc.2010.05.006. 
Potts, D. M. and Addenbrooke, T. I. (1997). "A structure's influence on tunnelling-induced ground movements.” Proc. ICE - Geotech. Eng., 125(2), 109-125. doi:10.1680/igeng.1997.29233.

Rampello, S., Callisto, L., Viggiani, G., and Soccodato, F. M. (2012). "Evaluating the effects of tunnelling on historical buildings: the example of a new subway in Rome / Auswertung der Auswirkungen des Tunnelbaus auf historische Gebäude am Beispiel einer neuen U-Bahnlinie in Rom." Geomech. Tunn., 5(3), 275-299. doi:10.1002/geot.201200017.

Ritter, S., Giardina, G. G., Franza, A., and DeJong, M. J. (2020). "Building Deformation Caused by Tunneling: Centrifuge Modeling." J. Geotech. Geoenvironmental Eng., 146(5), 04020017. doi:10.1061/(ASCE)GT.1943-5606.0002223.

Son, M. and Cording, E. J. (2005). "Estimation of building damage due to excavation-induced ground movements." J. Geotech. Geoenvironmental Eng., 131(2), 162-177. doi:10.1061/(ASCE)10900241(2005)131:2(162).

Visone, C. (2008). "Performance-based approach in seismic design of embedded retaining walls." Ph.D. thesis, Univ. Naples Federico II, Italy.

Xu, J., Franza, A., and Marshall, A. M. (2020a). "Response of framed buildings on raft foundations to tunneling." J. Geotech. Geoenvironmental Eng., 146(11), 04020120. doi:10.1061/(ASCE)GT.19435606.0002376 .

Xu, J., Franza, A., Marshall, A. M., Losacco, N., and Boldini, D. (2020b). "Tunnel-framed building interaction: comparison between raft and separate footing foundations." Géotechnique (In Press. doi:10.1680/jgeot.19.P.393.

Yiu, W. N., Burd, H. J., and Martin, C. M. (2017). "Finite-element modelling for the assessment of tunnelinduced damage to a masonry building." Géotechnique, 67(9), 780-794. doi:10.1680/jgeot.sip17.P.249.

Zhao, Y. (2008). "In situ soil testing for foundation performance prediction." Ph.D. Thesis, Cambridge Univ. 


\section{List of Figures}

1 Building deformation parameters inferred from bay corner displacements. . . . . . 24

2 Experimental layout for different tunnel-frame configurations. . . . . . . . . . 25

3 Settlements of the raft foundations and underlying soil at $V_{l, t}=1 \%$ (left column: centrifuge data; right column: numerical results) . . . . . . . . . . . . . . 26

4 Settlements of the separate footings and underlying soil at $V_{l, t}=1 \%$ (left column: centrifuge data; right column: numerical results) . . . . . . . . . . . . . . . 27

5 Horizontal displacements of the separate footings and underlying soil at $V_{l, t}=1 \%$ (left column: centrifuge data; right column: numerical results) . . . . . . . . . . . 28

6 Maximum frame distortion for rafts (upper row: centrifuge; lower row: numerical). 29

7 Maximum frame distortion for separate footings: (a) centrifuge; (b) numerical predictions. . . . . . . . . . . . . . . . . . 30

8 Comparison of ASRE Class A (top) and C (bottom) predictions with centrifuge results for frame F2t3b6L: settlements $(\mathrm{a}, \mathrm{b})$ and angular distortions $(\mathrm{c}, \mathrm{d}) . \ldots$. . . . . 31

9 Modification factor of angular distortion for rafts: (a) central and (b) eccentric tunnels (envelopes from Xu et al. (2020a)). . . . . . . . . . . . . . . 32

10 Modification factor of angular distortion for footings: (a) central and (b) eccentric tunnels (envelopes from Xu et al. (2020b)) . . . . . . . . . . . . . . . 33

11 Modification factor of horizontal strains at the footings obtained from numerical models: (a) tensile and (b) compressive strains for central structures; (c) tensile and (d) compressive strains for eccentric tunnels. . . . . . . . . . . . . . . . . 34 


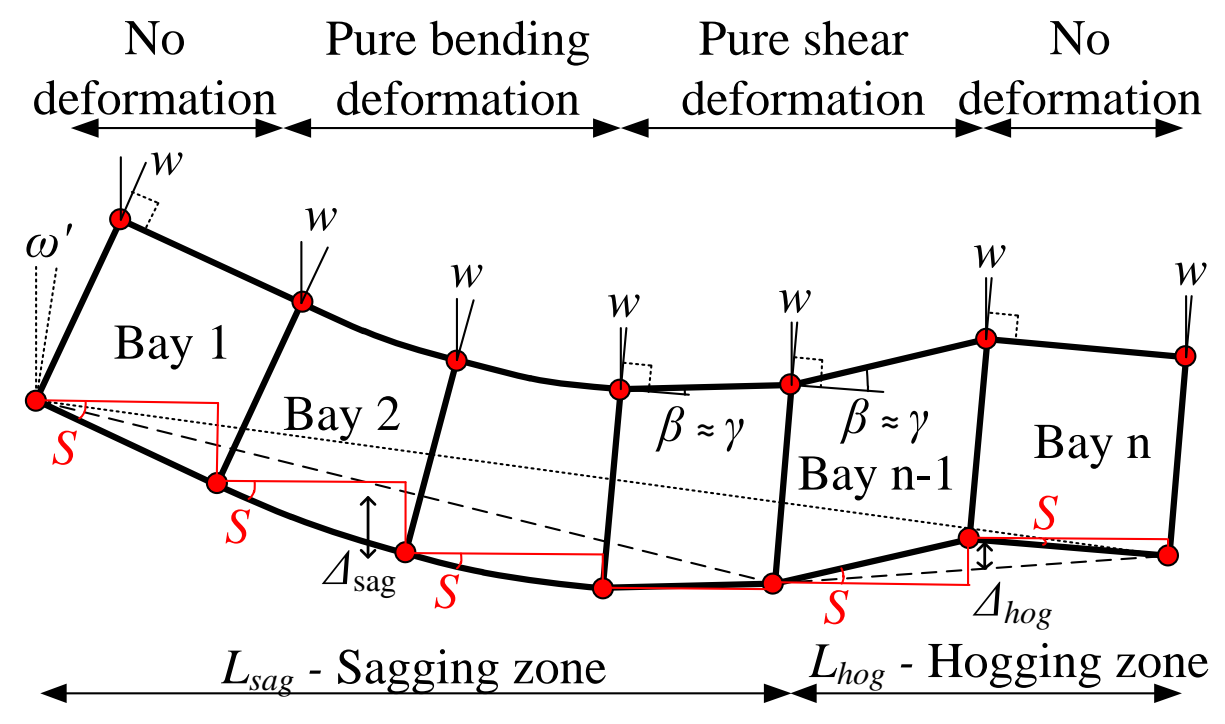

Fig. 1. Building deformation parameters inferred from bay corner displacements. 


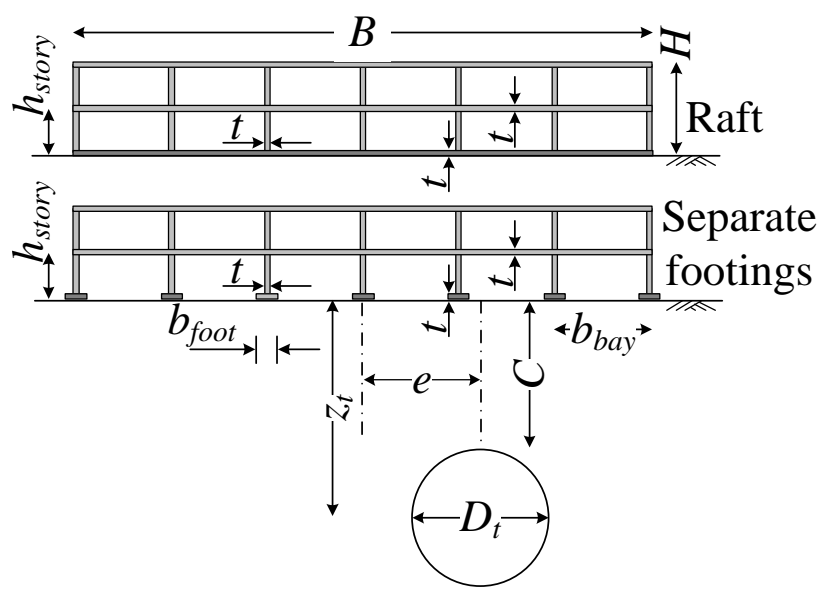

Fig. 2. Experimental layout for different tunnel-frame configurations. 


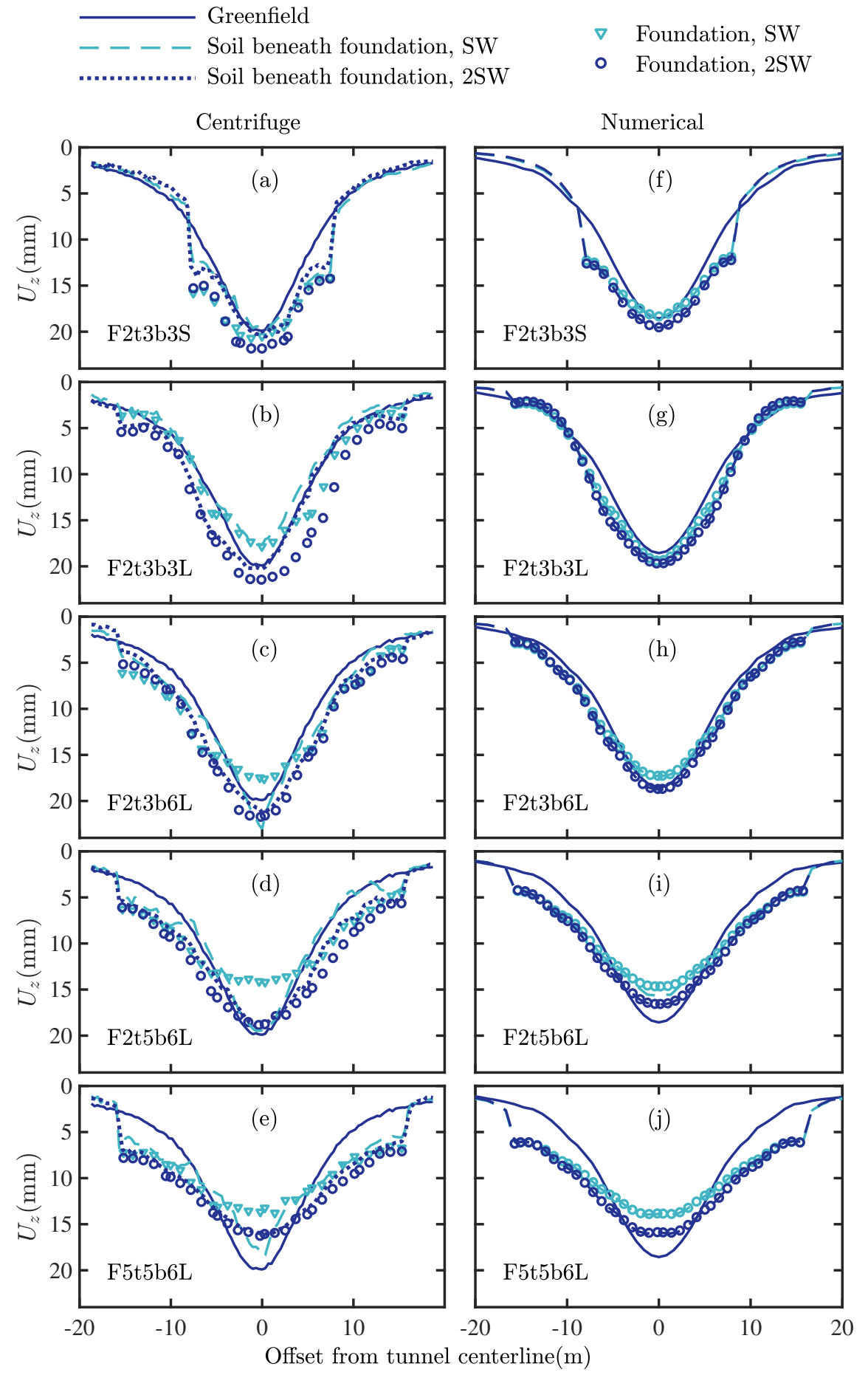

Fig. 3. Settlements of the raft foundations and underlying soil at $V_{l, t}=1 \%$ (left column: centrifuge data; right column: numerical results). 


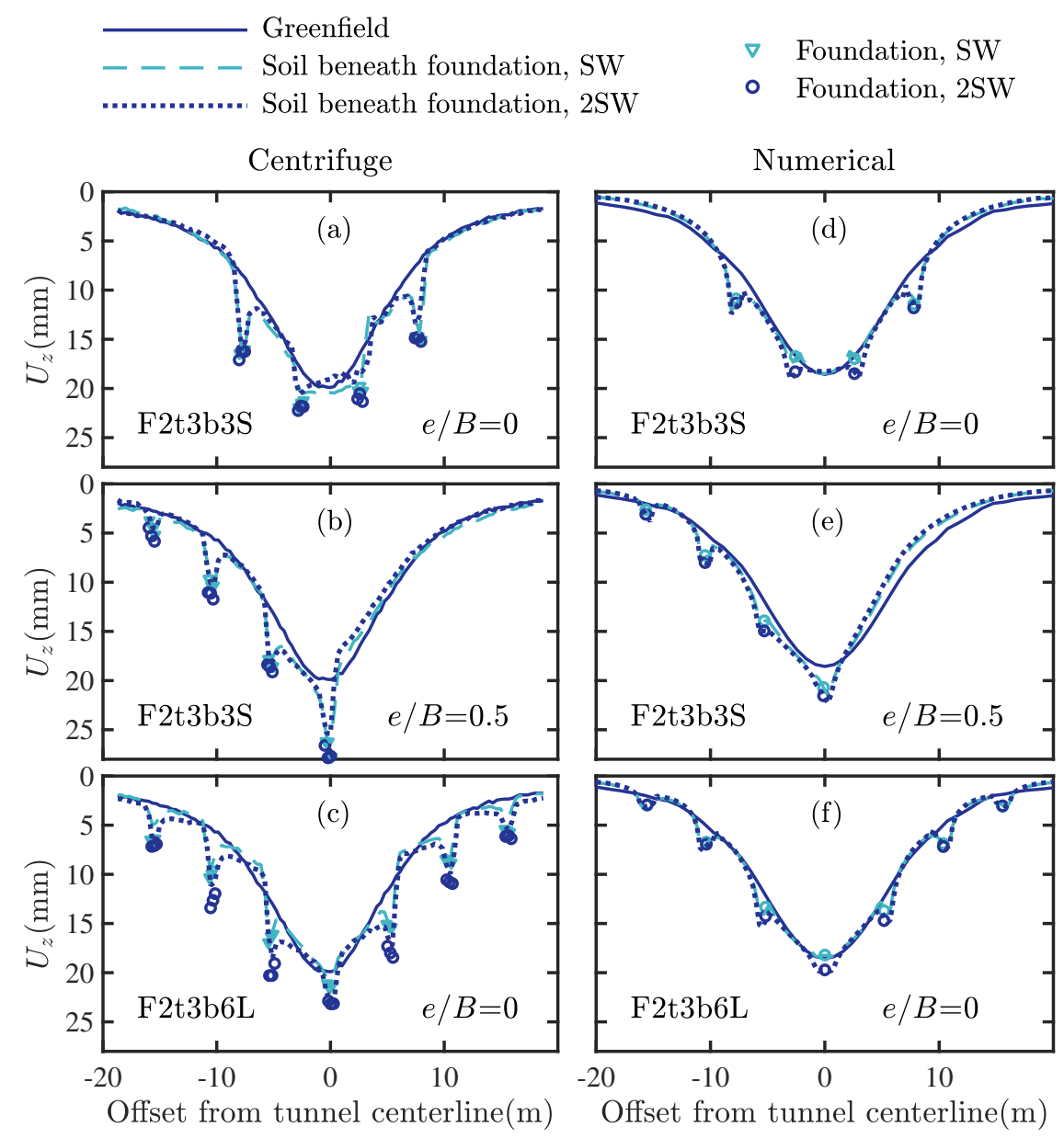

Fig. 4. Settlements of the separate footings and underlying soil at $V_{l, t}=1 \%$ (left column: centrifuge data; right column: numerical results). 


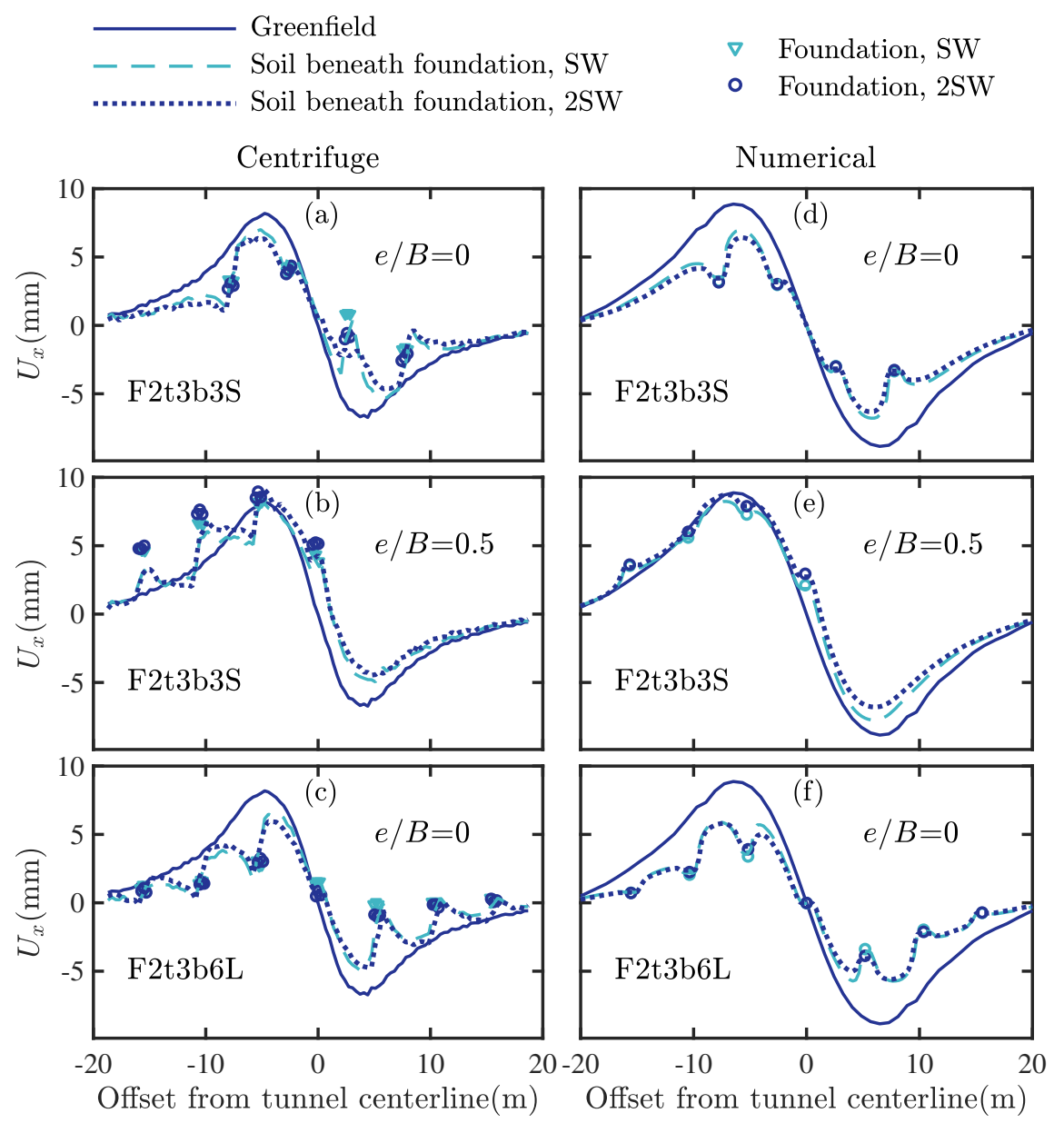

Fig. 5. Horizontal displacements of the separate footings and underlying soil at $V_{l, t}=1 \%$ (left column: centrifuge data; right column: numerical results). 

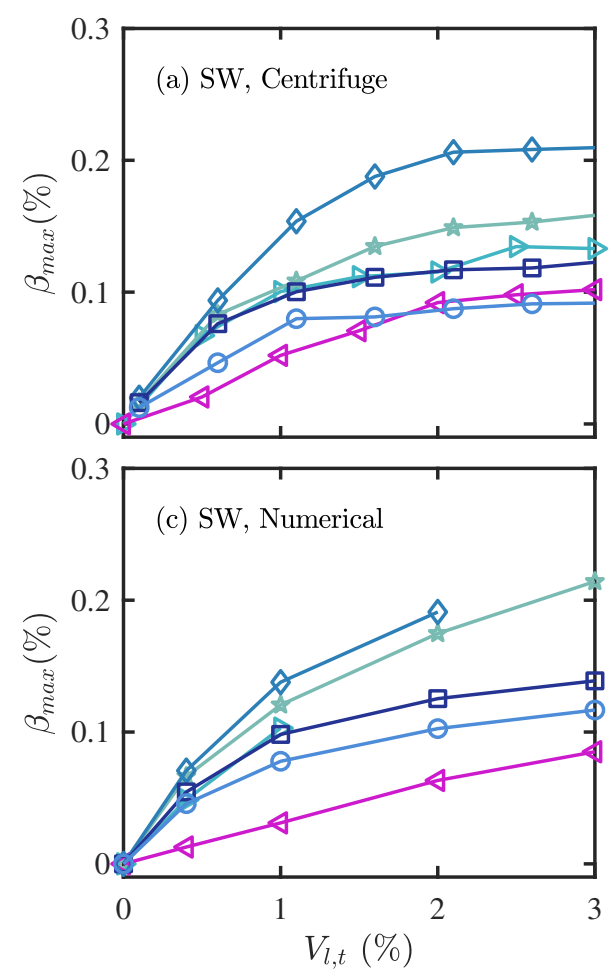
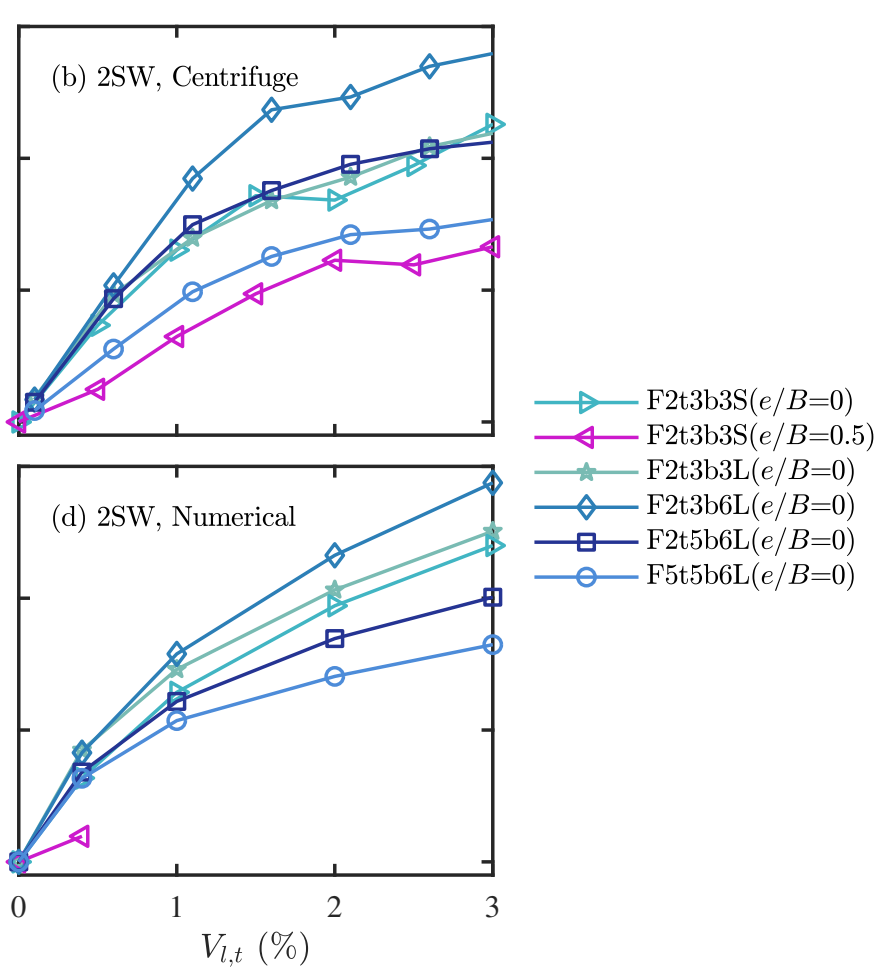

Fig. 6. Maximum frame distortion for rafts (upper row: centrifuge; lower row: numerical). 

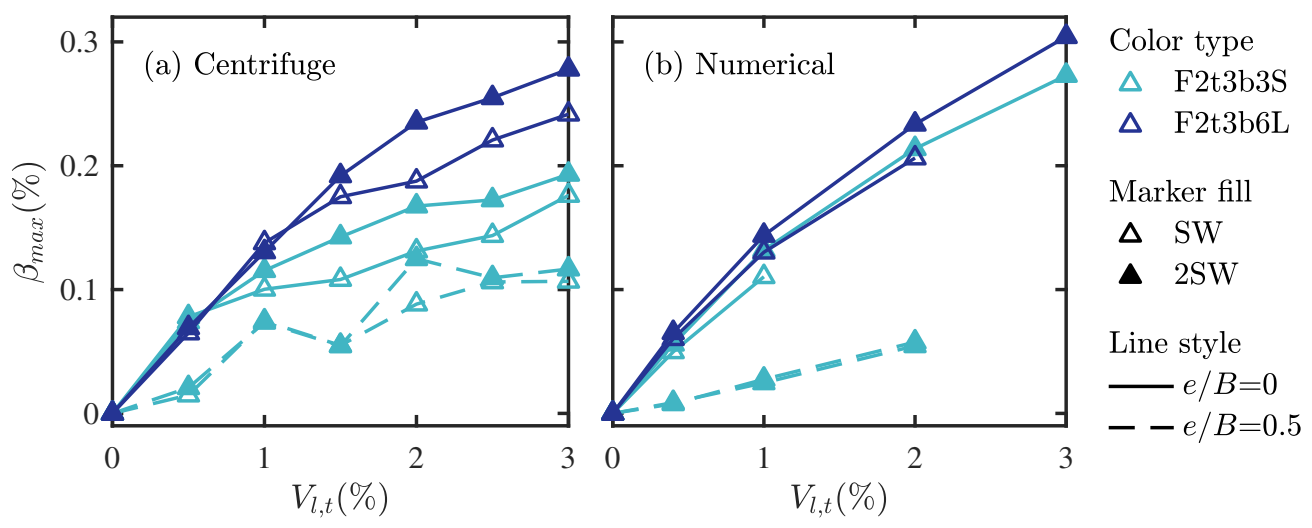

Fig. 7. Maximum frame distortion for separate footings: (a) centrifuge; (b) numerical predictions. 
Class A
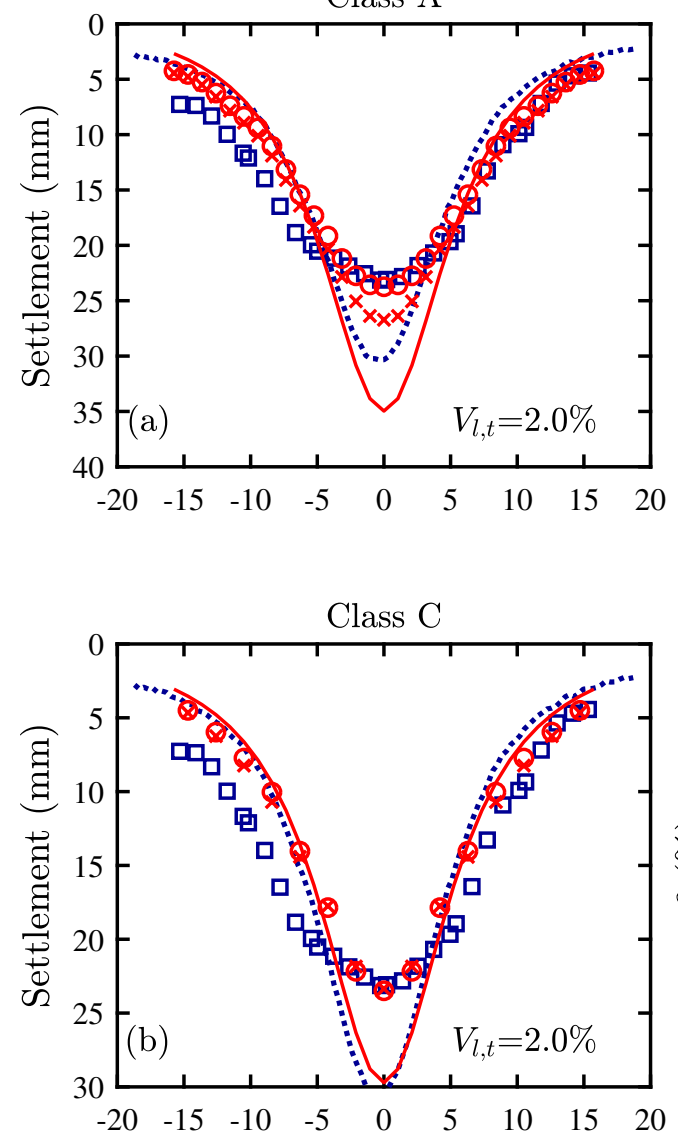

Offset from the tunnel centerline $(\mathrm{m})$
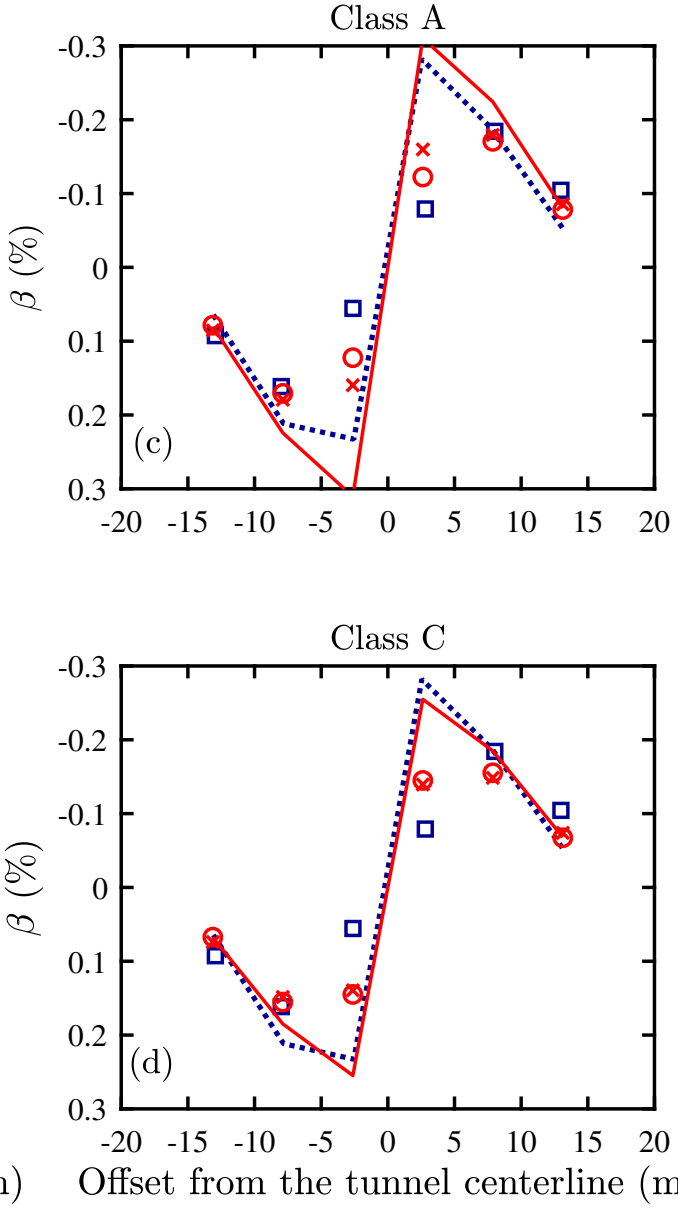

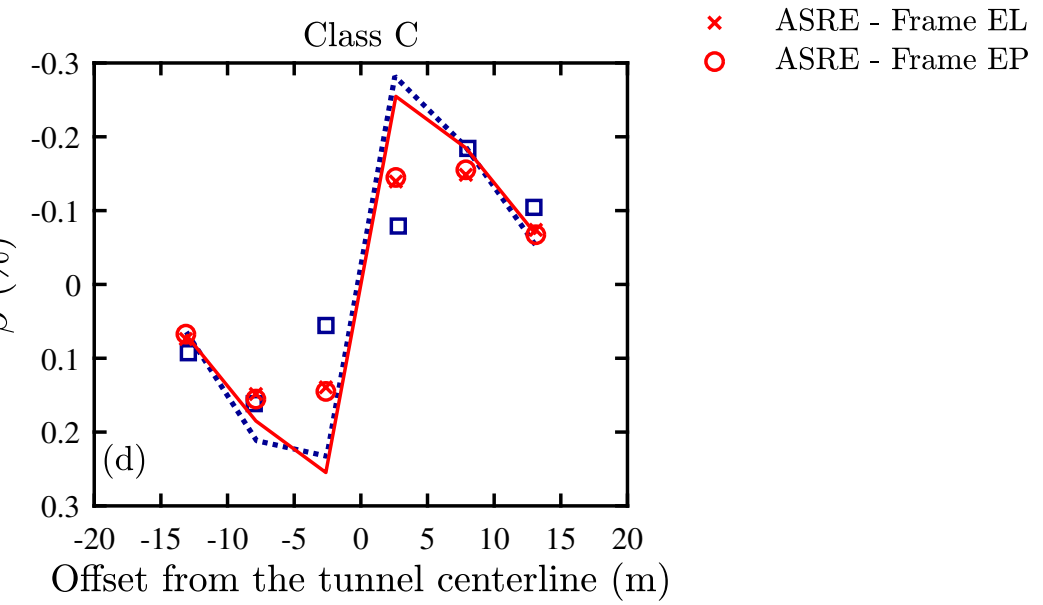

Fig. 8. Comparison of ASRE Class A (top) and C (bottom) predictions with centrifuge results for frame F2t3b6L: settlements (a,b) and angular distortions (c,d). 

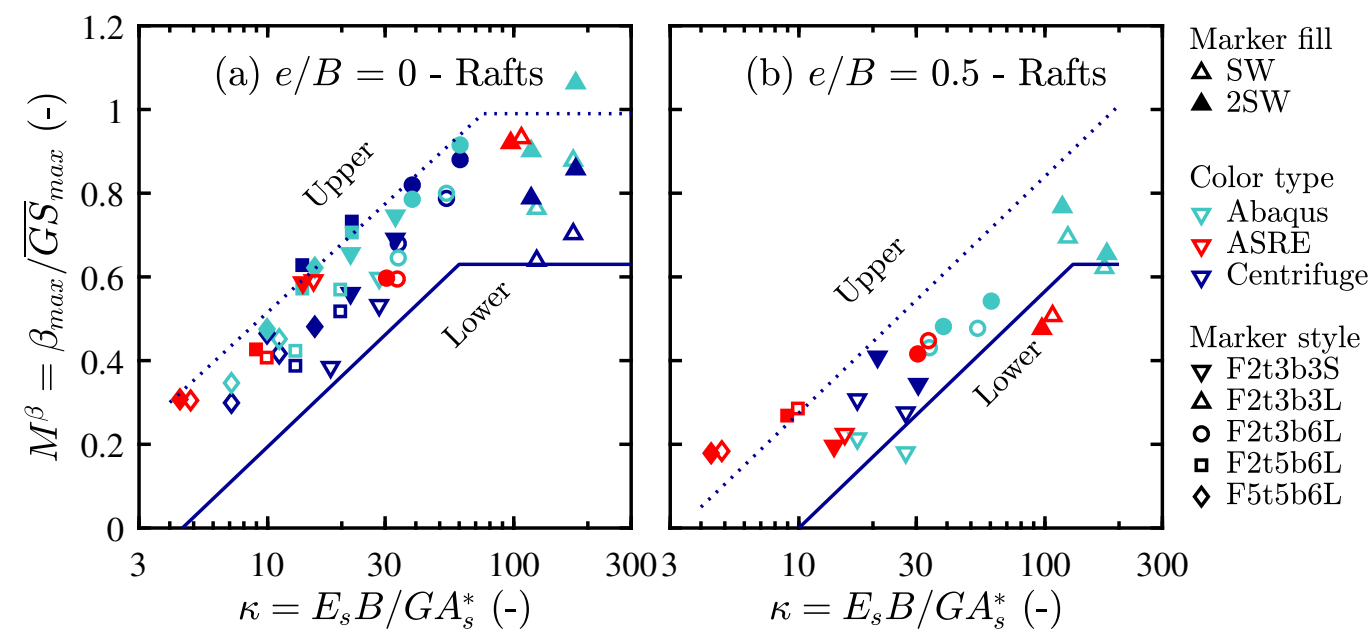

Fig. 9. Modification factor of angular distortion for rafts: (a) central and (b) eccentric tunnels (envelopes from Xu et al. (2020a)). 


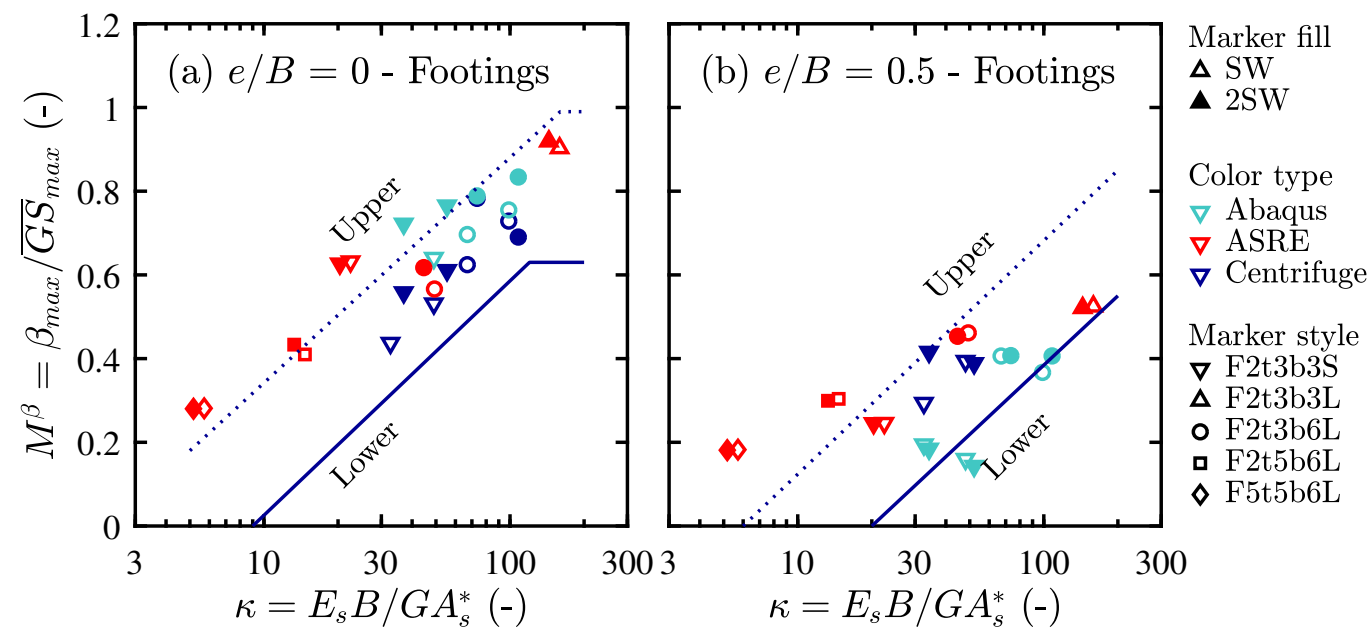

Fig. 10. Modification factor of angular distortion for footings: (a) central and (b) eccentric tunnels (envelopes from Xu et al. (2020b)). 


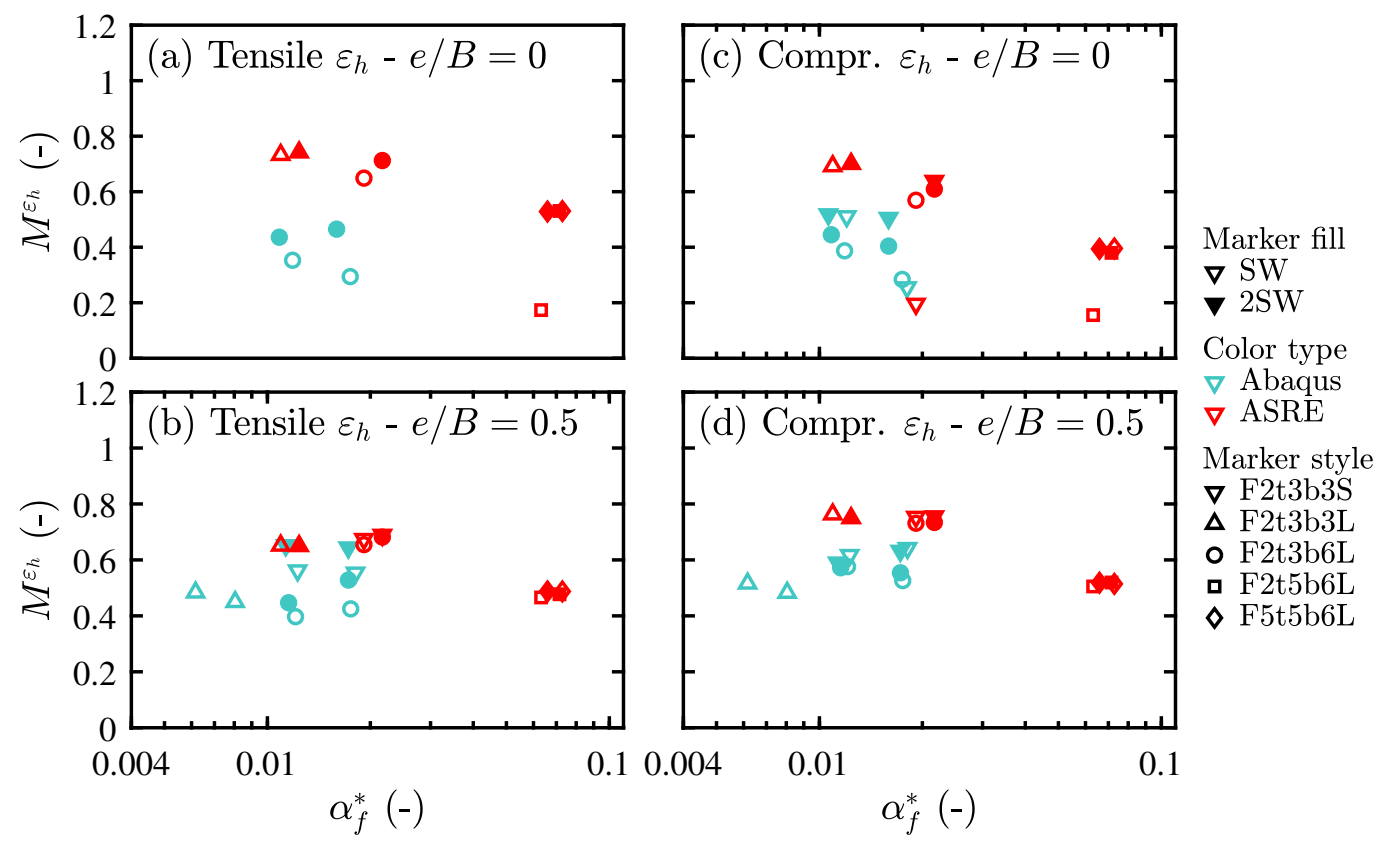

Fig. 11. Modification factor of horizontal strains at the footings obtained from numerical models: (a) tensile and (b) compressive strains for central structures; (c) tensile and (d) compressive strains for eccentric tunnels. 
List of Tables

637

1 Configuration of numerically simulated centrifuge tests. . . . . . . . . . 36 
TABLE 1. Configuration of numerically simulated centrifuge tests.

\begin{tabular}{lcccccccccccc}
\hline Label & Found. type & $\#$ & $\#$ & \multicolumn{4}{c}{ Centrifuge scale (dimension in mm) } & \multicolumn{4}{c}{ Prototype (dimension in m) } \\
& & stories & bays & $t$ & $H$ & $B$ & $b_{\text {bay }}$ & $t$ & $H$ & $B$ & $b_{\text {bay }}$ & $e / B$ \\
\hline F5t5b6L & Raft & 5 & 6 & 4.8 & 195.3 & 462.0 & 76.2 & 0.32 & 13.3 & 31.4 & 5.2 & 0 \\
F2t5b6L & Raft & 2 & 6 & 4.8 & 81.0 & 462.0 & 76.2 & 0.32 & 5.5 & 31.4 & 5.2 & 0 \\
F2t3b6L & Raft \& Sep. foot. & 2 & 6 & 3.2 & 79.4 & 460.4 & 76.2 & 0.22 & 5.4 & 31.3 & 5.2 & 0 \\
F2t3b3L & Raft & 2 & 3 & 3.2 & 79.4 & 460.4 & 152.4 & 0.22 & 5.4 & 31.3 & 10.4 & 0 \\
F2t3b3S & Raft \& Sep. foot. & 2 & 3 & 3.2 & 79.4 & 231.8 & 76.2 & 0.22 & 5.4 & 15.8 & 5.2 & $0 ; 0.5$ \\
\hline
\end{tabular}

Note: $h_{\text {story }}=38.1 \mathrm{~mm}$ at model scale and $2.6 \mathrm{~m}$ at prototype for all frames. For separate footings, $b_{f o o t}=12 \mathrm{~mm}$ at model scale and $0.8 \mathrm{~m}$ at prototype. All configurations modeled for standard ( $\mathrm{SW}$ ) and double self-weight (2SW). 